\title{
Treatment with camu camu (Myrciaria dubia) prevents obesity by altering the gut microbiota and increasing energy expenditure in diet-induced obese mice
}

\author{
Fernando F Anhê, ${ }^{1,2}$ Renato T Nachbar, ${ }^{1}$ Thibault V Varin, ${ }^{2}$ Jocelyn Trottier, ${ }^{3,4}$ \\ Stéphanie Dudonné, ${ }^{2}$ Mélanie Le Barz, ${ }_{1}^{1,2}$ Perrine Feutry, ${ }^{2}$ Geneviève Pilon, ${ }^{1,2}$ \\ Olivier Barbier, ${ }^{3,4}$ Yves Desjardins, ${ }^{2}$ Denis Roy, ${ }^{2}$ André Marette ${ }^{1,2}$
}

\begin{abstract}
- Additional material is published online only. To view, please visit the journal online (http://dx.doi.org/10.1136/ gutjnl-2017-315565).

'Department of Medicine, Faculty of Medicine, Cardiology Axis of the Québec Heart and Lung Institute, Laval University, Québec, Canada ${ }^{2}$ Institute of Nutrition and Functional Foods, Laval University, Québec, Canada ${ }^{3}$ Laboratory of Molecular Pharmacology, EndocrinologyNephrology Axis, CHU-Québec Research Centre, Québec, Canada

${ }^{4}$ Faculty of Pharmacy, Laval University, Québec, Canada
\end{abstract}

\section{Correspondence to} Dr André Marette, Cardiology Axis of the Québec Heart and Lung Institute, Laval University, Québec QC G1V 0A6, Canada; andre.marette@criucpq.ulaval.

Received 27 October 2017 Revised 10 July 2018 Accepted 12 July 2018 Published Online First 31 July 2018

Check for updates

(c) Author(s) (or their employer(s)) 2019. No commercial re-use. See rights and permissions. Published by BMJ.

To cite: Anhê FF, Nachbar RT, Varin TV, et al. Gut

2019:68:453-464

\begin{abstract}
Objective The consumption of fruits is strongly associated with better health and higher bacterial diversity in the gut microbiota (GM). Camu camu (Myrciaria dubia) is an Amazonian fruit with a unique phytochemical profile, strong antioxidant potential and purported anti-inflammatory potential.

Design By using metabolic tests coupled with 165 rRNA gene-based taxonomic profiling and faecal microbial transplantation (FMT), we have assessed the effect of a crude extract of camu camu (CC) on obesity and associated immunometabolic disorders in high fat/ high sucrose (HFHS)-fed mice.
\end{abstract}

Results Treatment of HFHS-fed mice with CC prevented weight gain, lowered fat accumulation and blunted metabolic inflammation and endotoxaemia. CC-treated mice displayed improved glucose tolerance and insulin sensitivity and were also fully protected against hepatic steatosis. These effects were linked to increased energy expenditure and upregulation of uncoupling protein 1 mRNA expression in the brown adipose tissue (BAT) of CC-treated mice, which strongly correlated with the mRNA expression of the membrane bile acid (BA) receptor TGR5. Moreover, CC-treated mice showed altered plasma BA pool size and composition and drastic changes in the GM (eg, bloom of Akkermansia muciniphila and a strong reduction of Lactobacillus). Germ-free (GF) mice reconstituted with the GM of CCtreated mice gained less weight and displayed higher energy expenditure than GF-mice colonised with the FM of HFHS controls.

Conclusion Our results show that $\mathrm{CC}$ prevents visceral and liver fat deposition through BAT activation and increased energy expenditure, a mechanism that is dependent on the GM and linked to major changes in the BA pool size and composition.

\section{INTRODUCTION}

The prevalence of obesity and overweight is extremely high worldwide ${ }^{1}$ and worrisome predictions indicate that more than 1 billion people will be obese by $2030 .^{2}$ Visceral obesity is crucial for the development of metabolic complications of chronic inflammatory aetiology, such as type 2 diabetes, hepatic steatosis and

\section{Significance of this study}

What is already known on this subject?

- The consumption of fruits and vegetables is strongly associated with better health status and lower prevalence of obesity.

- Dietary intake of polyphenol-rich foods is importantly correlated with higher bacterial richness and diversity in humans, and animal studies have shown a clear association between the metabolic benefits of dietary polyphenols and important changes in the gut microbiota.

- While causation needs to be ascertained with respect to the role of the gut microbiota in the metabolic benefits of dietary phytochemicals, the mechanisms underlying the effects of these compounds on body weight and gut microbiota warrant further investigation.

What are the new findings?

- By providing a nutritionally relevant dose of a unique mix of phytochemicals, camu camu (CC) prevented obesity and metabolic syndrome in diet-induced obese mice through increasing energy expenditure.

- CC prevented metabolic endotoxaemia and reshaped the gut microbiota by drastically decreasing the abundance of Lactobacillus spp and promoting expansion of Barnesiella spp, Turicibacter spp and Akkermansia muciniphila.

- CC reduced the levels of circulating bile acids and altered bile acid composition towards lower conjugated primary bile acids and higher unconjugated secondary bile acids.

- Colonisation of germ-free mice with the faecal microbiota of CC-treated mice partially and transiently recapitulated the metabolic benefits seen in conventional mice treated with CC.

cardiovascular diseases. ${ }^{3}$ Deciphering the mechanisms leading to obesity and its comorbidities is therefore of utmost importance to guide the search for novel treatments and preventive strategies. Previous works have demonstrated the role of the gut microbiota in 
Significance of this study

\section{How might it impact on clinical practice in the foreseeable future?}

- Our data promote the consumption of $\mathrm{CC}$ and other phytochemical-rich fruits as a safe and easily implementable nutritional strategy to trigger the expansion of $A$. muciniphila and other potentially beneficial bacteria in the gut microbiota to alleviate several detrimental features of the metabolic syndrome.

controlling host metabolism. ${ }^{45}$ While diet importantly influences gut microbial community structure,${ }^{6}$ metagenome-wide association studies have revealed that the consumption of fruits and other phytonutrient-rich foods are among the strongest factors explaining alterations in the faecal microbiota of humans. ${ }^{78}$ Accordingly, recent animal studies have shown that polyphenol-rich fruit extracts reduce body weight gain and alleviate insulin resistance in association with changes in the gut microbiota. ${ }^{9}{ }^{10}$ However, it remains unclear to what extent these gut microbial changes contribute to metabolic benefits.

Camu camu (Myrciaria dubia) is an Amazonian fruit deemed a 'superfruit' for its unique phytochemical profile ${ }^{11}$ and alleged potent antioxidant and anti-inflammatory activity. ${ }^{12}{ }^{13}$ Vitamin C is abundant in camu camu, and the concentration of flavonoids such as ellagic acid, ellagitannins and proanthocyanidins is also elevated in this fruit. ${ }^{12}{ }^{13}$ As camu camu is a prolific assembly of potentially healthy phytochemicals, we sought to analyse the impact of the administration of a crude extract of camu camu (which mimics well the totality of its phytonutrients at nutritionally relevant doses) on obesity and associated immunometabolic disorders in diet-induced obese mice. We also aimed at thoroughly investigating the role of the gut microbiota in mediating the effects of camu camu on host metabolism.

\section{MATERIALS AND METHODS}

\section{Animals}

C57Bl/6J male mice aged 8 weeks (Jackson, USA) were individually housed in a controlled environment (12 hours daylight cycle, lights off at 18:00 hours) with food and water ad libitum in the animal facility of the Institute of Nutrition and Functional Foods. After 2 weeks of acclimatisation on a normal-chow diet (Teklad 2018, Harlan), mice were randomly divided into four groups $(n=12)$ and fed on chow or a high-fat high-sucrose (HFHS) diet (see online supplementary table 1). Treatment started concomitantly with the introduction of HFHS diet and consisted of daily oral doses $(200 \mathrm{mg} / \mathrm{kg}$ ) of resuspended crude extract of camu camu (Sunfood, San Diego, USA), vitamin C $(6.6 \mathrm{mg} / \mathrm{kg})$ or the vehicle used to resuspend the extract and vitamin C (ie, animal facility's drinking water) throughout 8 weeks. The groups treated with the extract of camu camu and vitamin $C$ are referred to as $C C$ and $V i t C$, respectively throughout the manuscript, whereas vehicle-treated groups are identified as Chow and HFHS. The phytochemical profile of the camu camu extract is available in table 1 and online supplementary table 2. Body weight gain and food intake were assessed twice a week. At week 8, animals were anaesthetised in chambers saturated with isoflurane and then sacrificed by cardiac puncture. Blood was drawn in tubes containing 2 IU of heparin and immediately centrifuged in order to separate plasma from cells. Brown, subcutaneous and visceral fat pads were carefully collected along with gastrocnemius muscle, liver, intestines, pancreas and heart.
Table 1 Chemical characterisation of the camu camu extract

\begin{tabular}{|c|c|c|}
\hline & $\begin{array}{l}\text { Extract content } \\
\text { (mg/100 g dry } \\
\text { weight) }\end{array}$ & $\begin{array}{l}\text { Daily intake* } \\
\text { (mg/kg body } \\
\text { weight) }\end{array}$ \\
\hline Total polyphenols & $6550 \pm 140$ & 13.10 \\
\hline Anthocyanins & nd & \\
\hline Proanthocyanidins & $1854.97 \pm 6.53$ & 3.7 \\
\hline Flavanols/flavonols & $110.8 \pm 10$ & 0.22 \\
\hline Quercetin & $33.5 \pm 1.25$ & 0.06 \\
\hline Quercetin-glucoside & $5.6 \pm 0.51$ & 0.01 \\
\hline Quercetin-galactoside & $2.0 \pm 0.83$ & 0.004 \\
\hline Quercetion-3-xyloside & $3.5 \pm 0.96$ & 0.007 \\
\hline Quercetion-3-arabinoside & $0.7 \pm 0.39$ & 0.001 \\
\hline Myricetin & $74.3 \pm 1.96$ & 0.14 \\
\hline Myricetin-glucoside/galactoside & $8.4 \pm 1.63$ & 0.01 \\
\hline Coumaroyl-glucosides & $1.0 \pm 0.26$ & 0.002 \\
\hline Phenolic acids & $100.0 \pm 0.001$ & 0.20 \\
\hline 4-Hydroxybenzoic acid & $1.1 \pm 0.31$ & 0.002 \\
\hline p-Coumaric acid & $4.6 \pm 0.30$ & 0.009 \\
\hline Ferulic acid & $0.3 \pm 0.03$ & 0.0006 \\
\hline Protocatechuic acid & $0.4 \pm 0.12$ & 0.0008 \\
\hline Gentisic acid & $0.2 \pm 0.12$ & 0.0004 \\
\hline Ellagitannins & $450.0 \pm 110$ & 0.90 \\
\hline Ellagic acid & $44.0 \pm 0.001$ & 0.08 \\
\hline Casuarictin & $7.0 \pm 0.14$ & 0.01 \\
\hline Ellagitannin B & $117.1 \pm 6.70$ & 0.23 \\
\hline Ellagic acid & $44.0 \pm 0.001$ & 0.08 \\
\hline Gallic acid & $26.1 \pm 0.76$ & 0.05 \\
\hline \multicolumn{3}{|l|}{ Sugar } \\
\hline Glucose & $1110 \pm 0.08$ & 2.22 \\
\hline Fructose & $1790 \pm 0.11$ & 3.58 \\
\hline Polysaccharides & $7280 \pm 5.04$ & 14.56 \\
\hline Fibres & $34290 \pm 685.8$ & 68.58 \\
\hline Insoluble & $8092.44 \pm 161.8$ & 16.18 \\
\hline Fibres & $3669.03 \pm 73.3$ & 7.33 \\
\hline Vitamin C & $3330 \pm 90.8$ & 6.66 \\
\hline
\end{tabular}

Data are presented as the mean \pm SD.

*Daily intake was calculated based on the $200 \mathrm{mg}$ of camu camu extract/kg of body weight dose orally given to mice for 8 weeks.

nd, non-detected.

\section{Metabolic cages}

At week 5, mice were individually placed in metabolic cages (Comprehensive Laboratory Animal Monitoring System, CLAMS, Columbus Instruments, Columbus, Ohio, USA). Room temperature for all metabolic studies was maintained at $28^{\circ} \mathrm{C}$ to keep mice at thermoneutrality ${ }^{1415}$ and with a 12-hour light/dark cycle. The relationship between metabolic rate and body mass was normalised by using the Kleiber's interspecific mass exponent (0.75) to calculate the metabolic body size (body weight $\left.{ }^{0.75}\right) \cdot{ }^{16} 17$

\section{Germ-free mice and faecal microbiota transplants}

In an additional cohort, faecal pellets of vehicle-treated and CC-treated conventional mice were harvested at week 6 and transferred to anaerobic chambers for resuspension in phosphate-buffered saline (PBS) $(110 \mathrm{mg}$ of faeces $/ 350 \mu \mathrm{L}$ of PBS). Fourteen germ-free $\mathrm{C} 57 \mathrm{Bl} / 6 \mathrm{~N}$ male mice aged 8 weeks were purchased from Taconic, USA, and, on the day of arrival, the sterile containers were opened under a germ-free laminar flow hood and mice were gavaged with freshly prepared faecal resuspensions $(350 \mu \mathrm{L})$. Germfree mice reconstituted with the faecal microbiota of vehicle-treated 
HFHS-fed mice are referred to as HFHS receivers $(n=7)$, whereas those reconstituted with the faecal slurry of CC-treated HFHS-fed mice are referred to as CC receivers $(n=7)$ throughout the manuscript. Receiver mice were initially housed in metabolic cages for 4 days (Columbus Instruments) and then transferred to ventilated sterile cages for 10 additional days and fed a low-fat diet (Research diets, D12450H) throughout the study.

The methods used to assess interscapular temperature, glucose homeostasis, plasma bile acids (BA), protein and gene expression and gut microbial profile are available in online supplementary material. Primer sequences are available in online supplementary table 3 .

\section{Statistical analysis}

Data are expressed as mean \pm SEM. One-way analysis of variance (ANOVA) with Bonferroni post hoc test (for parametric data sets) or Kruskal-Wallis test with Dunn's multiple comparison test (for non-parametric data sets) were used to assign significance to the differences between groups (GraphPad Prism, USA). Two-way repeated measures ANOVA with a Bonferroni post-test (Sigmaplot, USA) was used when time was considered as a variable. All results were considered statistically significant at $\mathrm{p}<0.05$.

The statistical significance of differentially abundant and biologically relevant taxonomical biomarkers between two distinct biological conditions was measured using a linear discriminant analysis effect size (LEfSe).

\section{RESULTS}

\section{CC, but not vitamin C, prevents obesity and liver steatosis in} diet-induced obese mice

Daily oral administration of CC prevented diet-induced weight gain from day 14 onwards (figure 1A, B), and these findings were not related to changes in energy intake (figure 1C). CC prevented fat accretion in all fat depots, including visceral (ie, epididymal, retroperitoneal and mesenteric), subcutaneous (ie, inguinal) and interscapular brown fat (figure 1D). Intestinal length and the weight of gastrocnemius, pancreas, heart and caecum content were not affected by CC administration (see online supplementary figures $1 \mathrm{a}-\mathrm{f})$. Vitamin $\mathrm{C}$ did not prevent diet-induced obesity (figure $1 \mathrm{~A}-\mathrm{D}$ ), suggesting that other constituents in the extract are responsible for the observed anti-obesity effects. Liver weight was slightly, yet not significantly, reduced in CC-treated mice (figure 1E), whereas diet-induced hepatic steatosis and dyslipidemia were fully prevented by CC treatment, as indicated by lower triglyceride accumulation in both liver and circulation of CC-treated mice versus HFHS control mice (figure 1F, G). We found no differences in daily faecal energy output between CC and HFHS mice, suggesting that CC did not alter food digestion or intestinal absorption (figure $1 \mathrm{H}$ ). CC increased energy expenditure and that was unrelated with augmented physical activity (figure 1I and online supplementary figures $1 \mathrm{~g}-1)$, resulting in less energy available for storage (figure $1 \mathrm{~J}$ ). In line with increased oxygen consumption, CC-treated mice showed a tendency $(p=0.08)$ towards higher interscapular temperature than vehicle-treated HFHS-fed mice (figure 1K, HFHS $35.66^{\circ} \mathrm{C} \pm 0.09$; $\mathrm{CC} 35.97^{\circ} \mathrm{C} \pm 0.08$ ). Taken together, these results show that $\mathrm{CC}$ prevents diet-induced weight gain and adiposity by increasing energy expenditure and thermogenesis.

\section{CC blunts adipose tissue inflammation, alleviates metabolic endotoxaemia and improves glucose homeostasis in diet- induced obese mice}

CC treatment prevented metabolic inflammation in the adipose tissue, leading to a strong tendency $(p=0.07)$ towards reduced interleukin-1 $\beta$ (IL-1 $\beta)$. While the levels of vascular endothelial growth factor (VEGF), IL-6, interferon- $\gamma$ (INF- $\gamma$ ) and regulated on activation normal $T$ cell expressed and secreted (RANTES) were not altered by $\mathrm{CC}$ treatment, we found lower levels of monocyte chemoattractant protein-1 (MCP-1) in CC-treated versus vehicle-treated HFHS-fed mice (figure 2A-F). In line with reduced MCP-1, a key orchestrator of macrophage infiltration in the adipose tissue, we found lower mRNA expression of Adgre (encodes for F4/80) and lower crown-like structure (CLS) density in the epididymal adipose tissue (eWAT) of CC mice (figure $2 \mathrm{G}$ and online supplementary figures $2 \mathrm{a}-\mathrm{c}, \mathrm{f})$. Consistent with improved adipose tissue inflammation, CC mice showed reduced adipocyte size (see online supplementary figures $2 \mathrm{a}-\mathrm{e})$. CC treatment also prevented metabolic endotoxaemia, as indicated by lower circulating levels of LPS (figure 2H).

While fasting glycaemia was not different between CC-treated and HFHS control mice (figure 2I), CC administration prevented fasting hyperinsulinaemia (figure 2J), resulting in improved fasting insulin resistance as suggested by lower HOMA-IR (figure 2K). Postglucose challenge, CC enhanced glucose clearance (figure $2 \mathrm{~L}, \mathrm{M}$ ) and limited diet-induced hyperinsulinaemia (figure $2 \mathrm{~N}, \mathrm{O}$ ), whereas insulin sensitivity $10 \mathrm{~min}$ after insulin injection (figure 2P) was improved in CC mice versus HFHS control mice. These results indicate that the preventive effect of CC on HFHS-induced obesity and visceral fat accumulation and inflammation resulted in improved glucose homeostasis and insulin sensitivity.

\section{CC administration prevents obesity-driven dysbiosis}

Faecal samples were harvested at the end of the study (ie, week 8 ) and at the end of the acclimatisation period on a Chow diet (baseline -bs) to verify the uniformity of the microbial profile at the study beginning. Principal coordinate analysis revealed that while all samples clustered similarly at baseline (when all mice were fed a standard chow diet), 8 weeks of HFHS feeding drastically changed gut microbial profile (figure 3A). CC-treated mice, however, clustered partially apart of vehicle-treated HFHS-fed mice samples, suggesting important changes in CC gut microbial profile (figure $3 \mathrm{~A}$ ). CC treatment fully prevented the diet-induced decrease in microbial richness (figure $3 \mathrm{~B}$ ) and the increase in the Firmicutes/Bacteroidetes ratio (figure 3C, D), two hallmarks of obesity-driven dysbiosis. ${ }^{18-20} \mathrm{CC}$ treatment prevented the HFHS-driven reduction in Bifidobacterium and Barnesiella (figure $3 \mathrm{E}$ and online supplementary figures 31, n) and reduced the relative abundance of Lactobacillus, the later explaining most of the reduction in Firmicutes found in the gut microbiota of CC versus HFHS (figure $3 \mathrm{E}$ and online supplementary figure 3a).

The LEfSe approach ranked Barnesiella spp and Turicibacter spp along with a major decrease in operational taxonomic units (OTUs) assigned to Lactobacillus spp as the main features discriminating faecal bacterial communities of CC mice from that of HFHS control mice (figure $3 \mathrm{G}$ and online supplementary figure $3 \mathrm{a}, \mathrm{k}, \mathrm{l}$ ). Higher abundace of Barnesiella and Turicibacter was also noticed in Chow mice as compared to vehicle-treated HFHS mice (figure 3F). Since we found a tendency towards expansion of Akkermansia muciniphila in CC mice (figure 3E and online supplementary figure $3 \mathrm{~m}$ ), we quantified this bacterium by $\mathrm{qPCR}$ and found a major increase in its abundance in CC mice (figure $3 \mathrm{H}$ ). Although OTUs assigned to the genera Delftia, Roseburia, Anaerostipes, Anaerotruncus and Parabacteroides, and to unclassified genera within the families Christensenellaceae and Erysipelotrichaceae, were identified by LEfSe as discriminative taxa between CC and HFHS control mice (figure 3G), their abundances were considerably low (figure 3E). We 
A

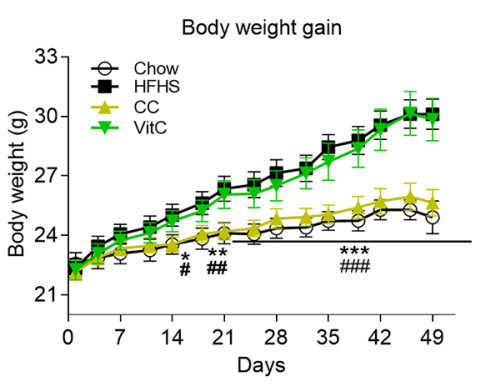

B

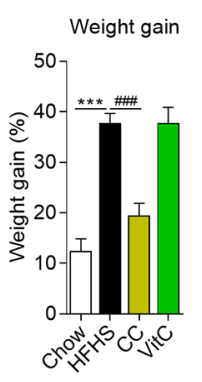

C

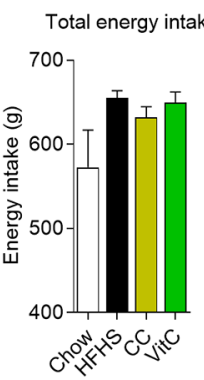

D

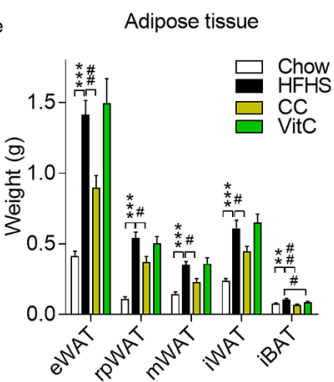

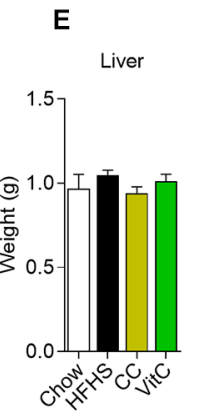

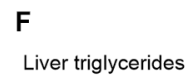

G

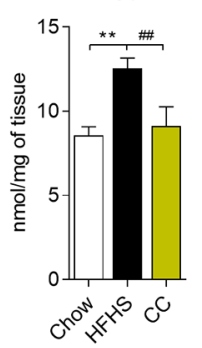

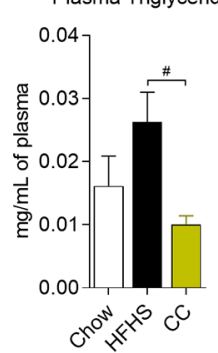

$\mathrm{H}$

Fecal energy excretion

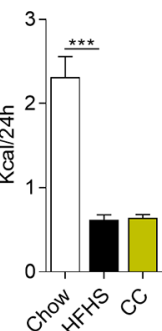

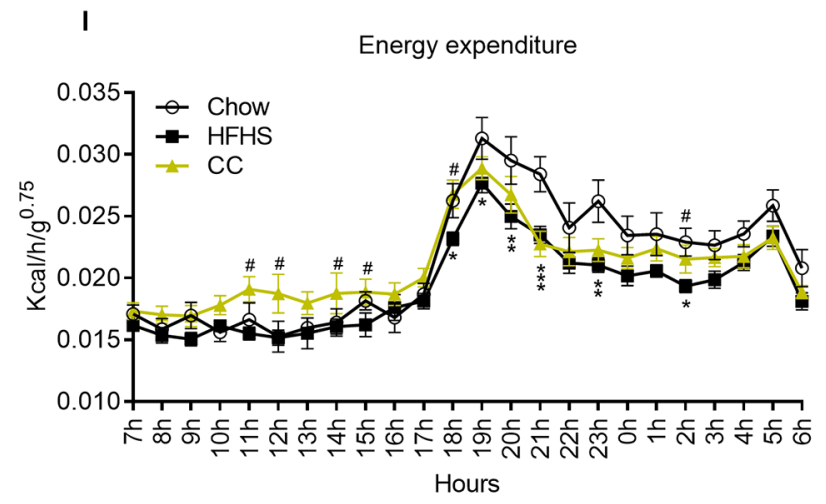

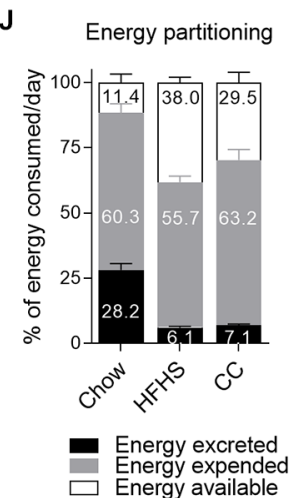

K Interscapular temperature

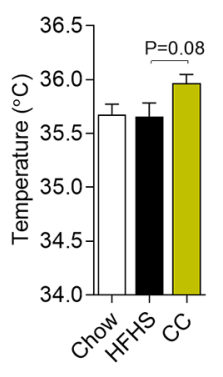

Figure 1 Camu camu (CC) administration, but not vitamin C (VitC), prevents diet-induced obesity. Mice were fed either a standard chow diet (Chow, $n=12)$ or a high fat/high sucrose (HFHS, $n=12)$ diet and treated with daily doses of an extract of CC $(n=12)$ or VitC $(n=12)$ throughout 8 weeks. (A, B) Body weight gain; (C) cumulative energy intake. (D) Epididymal (eWAT), retroperitoneal (rpWAT), mesenteric (mWAT) and inguinal (iWAT) white adipose tissues (WAT) along with interscapular brown adipose tissue (iBAT) were harvested and weighed during necropsies. (E) Liver weight; (F) hepatic triglyceride content; (G) circulating triglycerides (6-hour fasted mice). At week 5, mice were temporarily housed in metabolic cages and $(\mathrm{H})$ faecal energy output and $(\mathrm{I})$ energy expenditure were assessed. Mice were monitored over a 3-day period (1 day of acclimatisation followed by 2 days of measurements). The relationship between metabolic rate and body mass was normalised by using the metabolic body size (ie, body mass ${ }^{0.75}$ ). (J) Energy partitioning. (K) Interscapular temperature. Data are expressed as the mean \pm SEM. (A, I) Two-way repeated measures analysis of variance (ANOVA) with Bonferroni post hoc test. (B-H, K) One-way ANOVA with Bonferroni post hoc test. ${ }^{*} \mathrm{P}<0.05,{ }^{* *} \mathrm{p}<0.01$ and ${ }^{* * *} \mathrm{p}<0.001$ for Chow vs HFHS; $" p<0.05, " \#<0.01$ and ${ }^{\# \# \#} p<0.001$ for CC vs HFHS.

therefore decided to focus on the major CC-related effects, particularly on Barnesiella, Turicibacter, Bifidobacterium, A. muciniphila and Lactobacillus.

\section{CC administration alters the plasma bile acid pool and upregulates the mRNA expression of markers of brown fat activation and browning of white adipose tissue}

Since BA have been shown to regulate energy homeostasis and brown adipose tissue activity, ${ }^{21-23}$ we sought to evaluate whether changes in the BA pool could be linked to the effects seen in CC-treated mice. While the relative abundance of circulating tauro- $\alpha$-murocholic acid (T $\alpha \mathrm{MCA})$ and tauro- $\beta$-murocholic acid (T $\beta$ MCA) was lower in CC mice, the proportion of $\beta$-murocholic acid and $\omega$-murocholic acid ( $\omega \mathrm{MCA})$ were both higher in these mice (figure $4 \mathrm{~B}$ and online supplementary table $5)$. Furthermore, the relative abundance of chenodeoxycholic acid (CDCA), deoxycholic acid (DCA), hyodeoxycholic acid and ursodeoxycholic acid (UDCA) was higher in the plasma of CC mice versus HFHS control mice (figure $4 \mathrm{~B}$ and online supplementary table 5), findings that paralleled decreased circulating BA (figure 4A and online supplementary table 5) and increased proportion of secondary and of unconjugated BA in the plasma of CC mice (figure 4B, online supplementary table 5 and online supplementary figures $4 a-d)$. Faecal BA concentration was similar between vehicle-treated and CC-treated mice (figure 4A, 

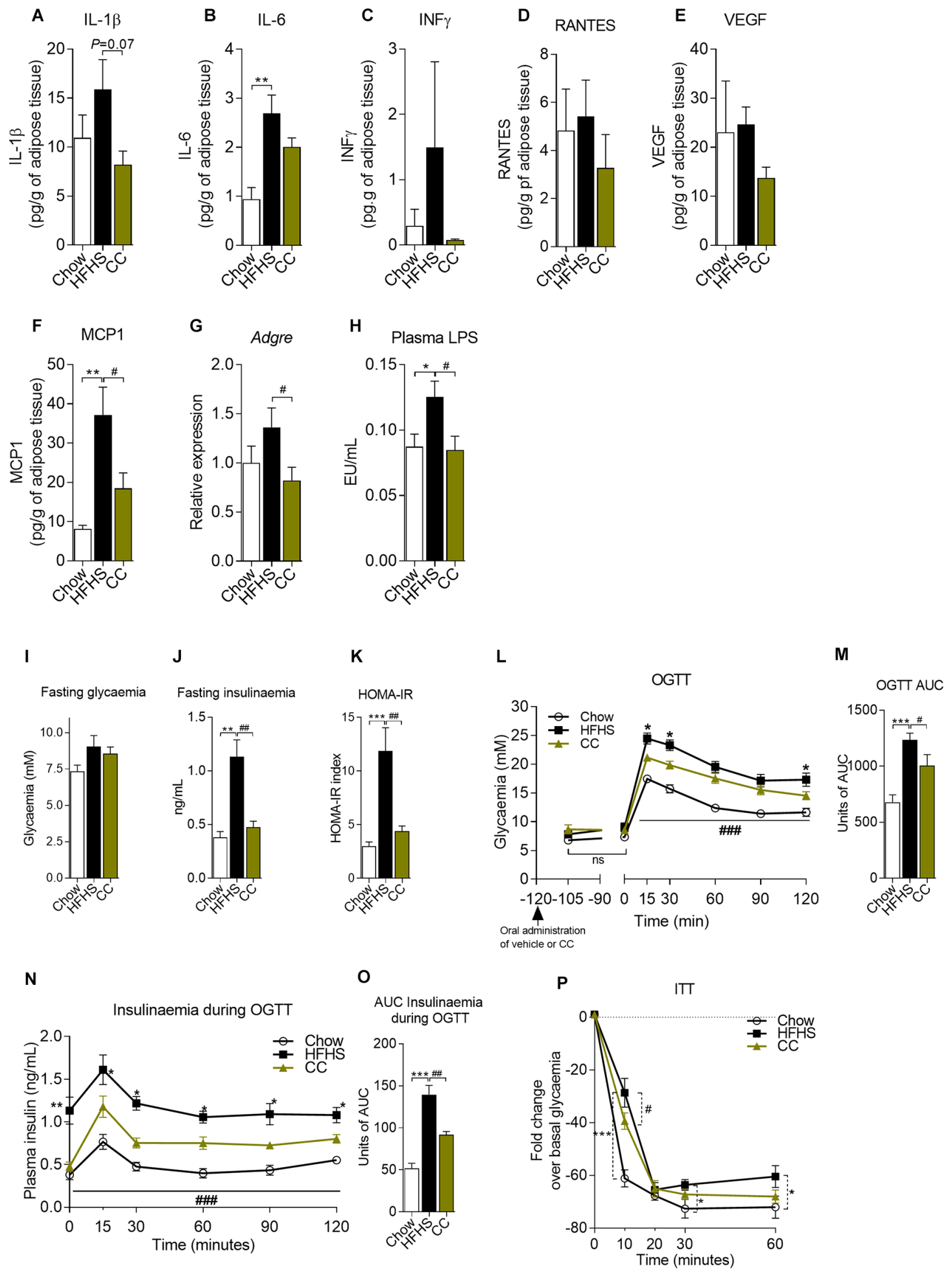

Figure 2 Camu camu (CC) blunts adipose tissue inflammation, alleviates metabolic endotoxaemia and improves glucose homeostasis in dietinduced obese mice. Protein lysates from epididymal fat pads (eWAT) of Chow $(n=12)$, high fat/high sucrose (HFHS) ( $n=12)$ and CC ( $n=12)$ were used to quantify (A) interleukin-1 $\beta$ (IL-1 $\beta)$, (B) interleukin-6 (IL-6), (C) interferon- $\gamma($ INF- $\gamma)$, (D) regulated on activation normal T cell expressed and secreted (RANTES), (E) vascular endothelial growth factor (VEGF) and (F) monocyte chemoattractant protein-1 (MCP-1). (G) qPCR analysis of Adgre (encodes for F4/80) mRNA expression in eWAT. (H) Circulating lipopolysaccharides (LPS) (6-hour fasted mice). At week 6, mice ( $n=12$ ) were fasted overnight (12 hours) and submitted to oral glucose tolerance tests (OGTT). CC was orally given 2 hours prior to the test, and glycaemia was assessed 15 min after CC administration (time point -105) to monitor possible changes in glycaemia associated with sugars present in CC. (I) Fasting glycaemia; (J) fasting insulinaemia; (K) homeostatic model assessment for insulin resistance (HOMA-IR); (L, M) OGTT. (N, O) Blood was collected during OGTT and used to assess insulinaemia after glucose challenge. At week 7, mice were fasted for 6 hours and (P) insulin tolerance tests (ITT) were carried out after intraperitoneal insulin injections $(0.65 \mathrm{IU} / \mathrm{kg})$. Data are expressed as the mean $\pm S E M$. $(\mathrm{L}, \mathrm{N}$ and $\mathrm{P})$ Two-way repeated measures analysis of variance (ANOVA) with Bonferroni post hoc test. (A-K, M and 0) One-way ANOVA with Bonferroni post hoc test. ${ }^{*} \mathrm{P}<0.05$, ${ }^{* *} \mathrm{p}<0.01$ and ${ }^{* * *} \mathrm{p}<0.001$ for Chow vs HFHS; ${ }^{\#}<0.05,{ }^{\#} \mathrm{p}<0.01$ and ${ }^{\# \#} \mathrm{p}<0.001$ for CC vs HFHS. AUC, area under the curve. 

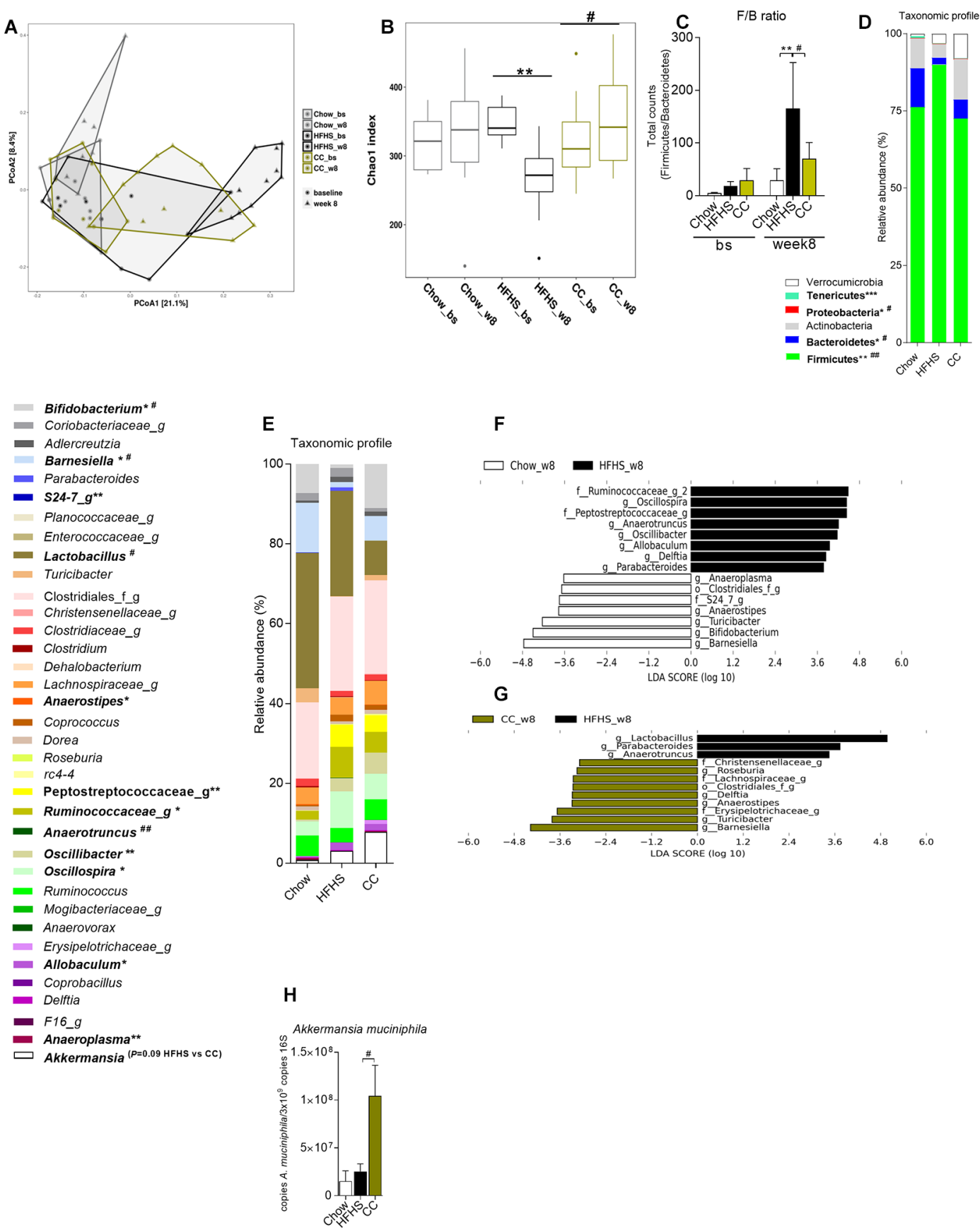

online supplementary figures $4 \mathrm{e}-\mathrm{h}$ and online supplementary tables 4 and 5), suggesting that altered circulating BA in CC mice was rather linked to differences in BA synthesis than excretion/ re-uptake. Consistent with reduced BA pool size, the ileal mRNA expression of $\mathrm{NrOb2}$ (encodes for Shp1) was downregulated in
CC mice; however, this was not sufficient to affect the mRNA expression of fibroblast growth factor 15 (Fgf15) and of other genes under farnesoid $\mathrm{X}$ receptor (FXR) control, such as the BA transporters organic solute transporter- $\alpha$ (Ost $\alpha$, Slc51a) and Ost $\beta$ (Slc51b), in the ileum of CC mice (figure 4C). These 


$$
\text { A }
$$

Bile Acids
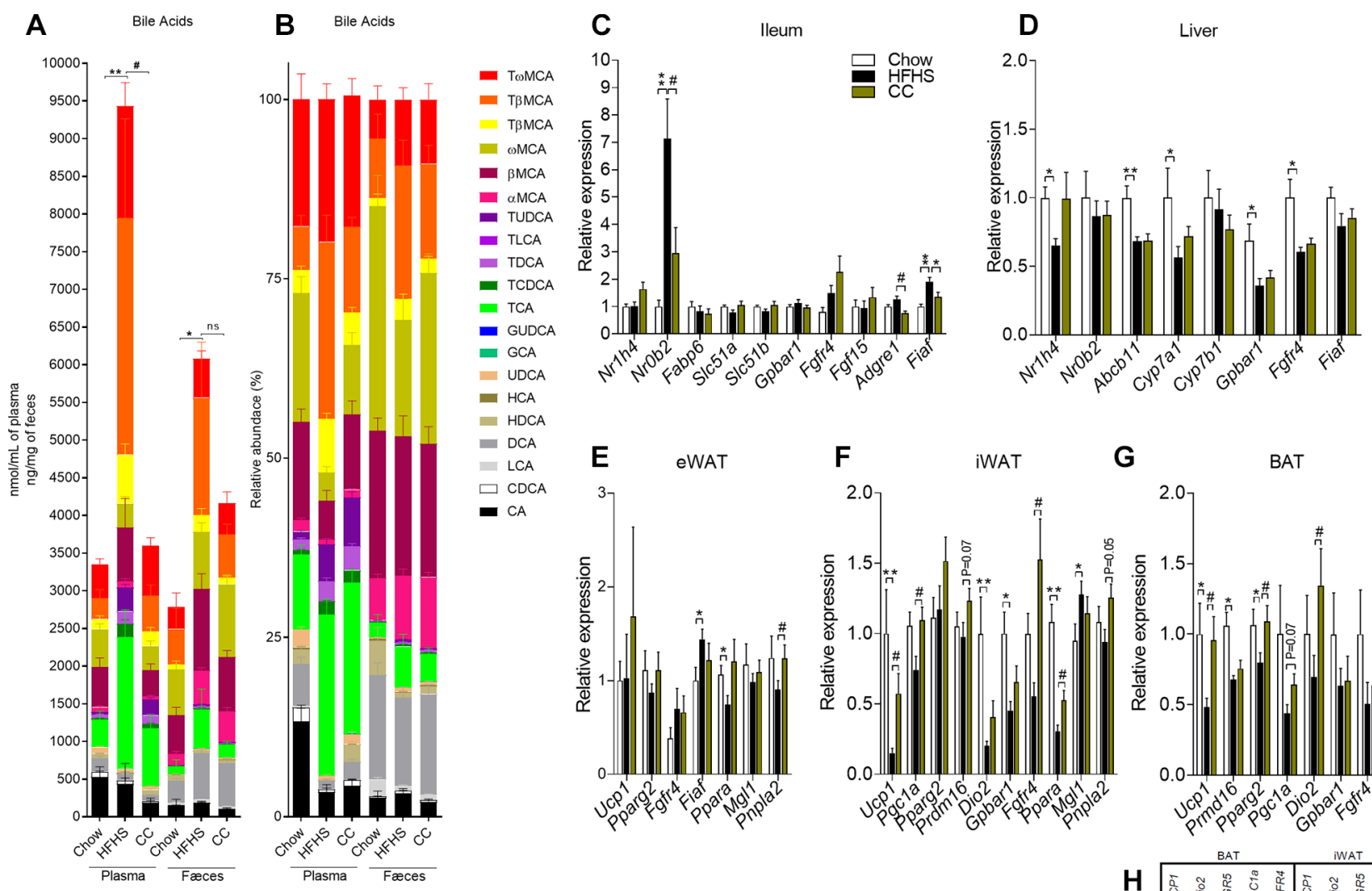

G

BAT

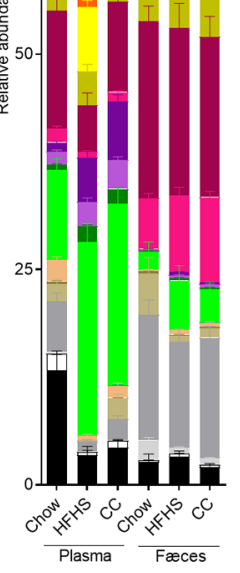

(20
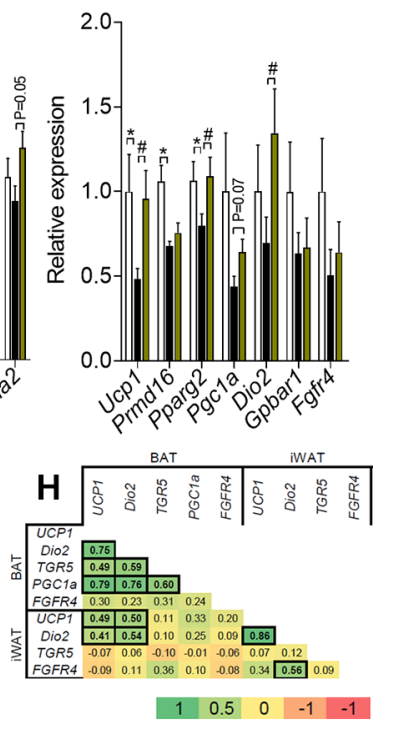

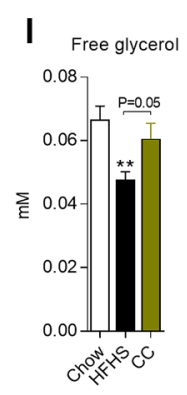

Figure 4 Camu camu (CC) administration alters bile acid pool size and composition and upregulates the mRNA expression of genes involved in brown adipose tissue activation, lipolysis and lipogenesis. (A, B) Plasma and faecal bile acids. Messenger RNA extracted from (C) ileum, (D) liver, (E) epididymal adipose tissue (eWAT), (F) inguinal adipose tissue (iWAT) and (G) brown adipose tissue (BAT) was quantified by RT-PCR. Relative expression was calculated using the $\triangle \Delta \mathrm{Ct}$ method with Chow mice as the group of reference and actin as the reference gene. $(\mathrm{H})$ Correlation matrix between BAT and iWAT mRNA expression profiles (bold values represent statistically significant correlations; $p=0.05$ if $0.404>r>0.404 ; p=0.01$ if $0.515>r>0.515 ; p=0.001$ if $0.628>r>0.628$ ). (I) Free glycerol. Data are expressed as the mean $\pm S E M$. One-way analysis of variance with Bonferroni post hoc test. ${ }^{*} \mathrm{P}<0.05$, ${ }^{* *} \mathrm{p}<0.01$ and ${ }^{* * *} \mathrm{p}<0.001$ for Chow vs HFHS; $\mathrm{*}<0.05$, ${ }^{\# \#} \mathrm{p}<0.01$ and ${ }^{\# \# \#} \mathrm{p}<0.001$ for $\mathrm{CC}$ vs HFHS.

findings were not related to changes in the mRNA expression of Cyp7a1 in the liver of CC mice in comparison with HFHS-fed control mice (figure 4D). Taken together, these results highlight drastic changes in the plasma BA pool size and composition in CC-treated mice.

Our findings revealed a significant upregulation in the mRNA levels of uncoupling protein 1 (Ucp1) and deiodinase 2 (Dio2) in both brown (BAT) and inguinal (iWAT) adipose tissues of CC-treated mice (figure 4F, G). Further supporting iWAT beiging, CC mice showed a tendency of higher peroxisome proliferator-activated receptor $\gamma$-2 (Pparg2) mRNA expression, increased PR domain containing 16 (Prdm16) gene expression and a tendency towards increased UCP1 protein expression in iWAT as compared with vehicle-treated HFHS-fed mice (figure 4F and online supplementary figure 5a). Concordant with higher BAT activity, the mRNA expression of Pparg2 was increased in the BAT of CC mice while PPAR $\gamma$ coactivator $1 \alpha(P g c 1 a)$ gene expression trended higher in the BAT of CC-treated versus vehicle-treated HFHS-fed mice (figure 4G). The mRNA expression of Fgfr 4 was upregulated in the inguinal fat of CC mice (figure 4F), but not in the eWAT or BAT of these mice (figure $4 \mathrm{E}, \mathrm{G}$ ). We found a strong correlation between Ucp1 and Dio2 mRNA expression in both iWAT and BAT, as well as between the mRNA expression of TGR5 (Gpbar1) and Ucp1 and Dio2 and PGC1a in BAT, all key genes involved in BAT activation (figure 4H). Our analysis also revealed that the mRNA expression of Fgfr 4 strongly correlated $(\mathrm{r}=0.86)$ with the mRNA expression of Dio2 in iWAT (figure $4 \mathrm{H}$ ). We did not detect significant changes in Ucp1 and Fgfr 4 mRNA expression in the eWAT (figure 4E). In summary, these data support increased brown fat activation coupled with browning of subcutaneous white adipose tissue. 
Lactobacillus spp, known to express bile salt hydrolases (BSH), were strongly correlated with several unconjugated BAs in the faeces. Akkermansia was negatively correlated with $\omega \mathrm{MCA}$ and positively correlated with the secondary BA LCA as well as the taurine-conjugated forms of DCA and UDCA in circulation (table 2). Other groups of bacteria, such as unclassified Erysipelotrichaceae and Lachnospiraceae as well as Barnesiella were also strongly correlated with both free and taurine conjugated forms of secondary BAs in plasma (table 2). These results suggest that key-phylotypes in the gut microbiota of CC mice may contribute to the synthesis of secondary BAs.

We next assessed whether changes in lipolytic/lipogenic program also contributes to reduced fat mass gain found in CC mice. In addition to higher plasma-free glycerol (figure 4I) and increased Ppara gene expression in both eWAT and iWAT of CC mice (figure 4F, G), we found increased mRNA expression of adipose triglyceride lipase (encoded by Pnpla2) in both eWAT and iWAT (figure 3E, F). Furthermore, while the ileal gene expression of the lipoprotein lipase (LPL) inhibitor fasting-induced adipose factor (Fiaf) was decreased in CC mice (figure 3C), the gene expression of monoacylglycerol lipase (Mgl1) was not affected by CC (figure 3E, F). While these findings point to increased lipolysis and fatty acid oxidation fuelled by higher triglyceride clearance, $\mathrm{Mgl1}$ is possibly poorly regulated at the transcriptional level. We found reduced protein expression of cluster of differentiation 36 (CD36) and fatty acid synthase (FAS) in the eWAT (see online supplementary figures $5 \mathrm{~h}, \mathrm{j})$ and lower fatty acid binding protein 4 (FABP4) and FAS in the iWAT of CC-treated mice (see online supplementary figures $5 \mathrm{~b}, \mathrm{f})$. The protein expression of CD36, PPAR $\gamma(-2$ and total) and acetyl-CoA carboxylase (ACC) in the iWAT was not affected by CC treatment (see online supplementary figures $\mathrm{c}-\mathrm{e})$, whereas protein levels of PPAR $\gamma$ ( -2 and total), FABP4 and ACC in the eWAT were not different between CC-treated and vehicle-treated HFHS-fed mice (see online supplementary figures $\mathrm{g}, \mathrm{i}, \mathrm{k})$. These data suggest that, in addition to higher energy expenditure, CC-mice display increased lipolysis/fatty acid oxidation and decreased lipid synthesis in both visceral and subcutaneous fat depots.

\section{Reconstitution of germ-free mice with the faecal microbiota of CC-treated mice increases energy expenditure}

We next performed faecal microbiota transplants (FMT) using germ-free mice colonised either with the faecal microbiota of CC-treated or vehicle-treated HFHS-fed donor mice. Throughout days 1, 2 and 3 postcolonisation, HFHS receivers gained, respectively, $3 \%, 2 \%$ and $2.4 \%$ of their initial body weight (figure 5A and online supplementary figure 6a). Conversely, CC receivers showed a rapid $5 \%$ body weight loss 1 day postcolonisation followed by a $3.7 \%$ and a $1.4 \%$ loss of their initial body weight on days 2 and 3 , respectively (figure $5 \mathrm{~A}$ ). This resulted in significant differences in weight gain between $\mathrm{CC}$ receivers and HFHS receivers on days $1(\mathrm{p}<0.001)$ and $2(\mathrm{p}=0.009)$ postcolonisation and a tendency $(\mathrm{p}=0.1)$ to be maintained at day 3 . There were no differences in energy intake or in faecal energy excretion between groups (figure 5B and online supplementary figures $6 \mathrm{~b}, \mathrm{c})$. Indirect calorimetry measurements performed throughout the initial days postcolonisation revealed a marked increase in energy expenditure unrelated to physical activity on days 1 and 2 postcolonisation. This effect was reduced on day 3 and completely lost on day 4 postcolonisation (figure 5C and online supplementary figures $6 \mathrm{~d}-\mathrm{i}$ ).
$16 \mathrm{~S}$ rRNA gene-based profiling revealed distinct gut microbial communities in HFHS-receiver and CC-receiver mice on day 1 postcolonisation (see online supplementary figure 6j). Microbial communities clustered further apart on day 2 postcolonisation, which was followed by higher similarity between HFHS-receiver and CC-receiver gut microbiota on day 3 postcolonisation (see online supplementary figure $6 \mathrm{k}, \mathrm{l}$ ). The differences seen on day 1 postcolonisation were mostly explained by a tendency of lower Firmicutes/Bacteroidetes ratio and higher presence of Verrucomicrobia (figure 5D, G and online supplementary table 6). The increased abundance of Verrucomicrobia in CC receivers was exacerbated on day 2 postcolonisation, reaching statistically significant levels, and lost on day 3 (figure $5 \mathrm{D}$ and online supplementary table 6). Since Verrucomicrobia is solely represented by A. muciniphila in the gut microbiota of mice and humans, the latter was as abundant as the former in CC receivers versus HFHS receivers during colonisation (figure $5 \mathrm{E}$ and online supplementary table 7). In addition to A. muciniphila, analysis at lower taxonomic level on day 1 postcolonisation revealed lower abundance of unclassified Lactobacillales and Lactobacillus and of Turicibacter in CC receivers versus HFHS receivers (figure 5E, F and online supplementary table 7). Barnesiella trended higher in $\mathrm{CC}$ receivers on days 1 and 2 postcolonisation (figure $5 \mathrm{E}, \mathrm{F}$ and online supplementary table 7 ). On day 2, CC receivers displayed higher proportions of rc4-4 (a genus within the Peptococcaceae family) and Bifidobacterium and lower abundance of Bacteroidales S24-7 and Allobaculum (figure 5E, F and online supplementary table 7). On day 3, increased dominance of Firmicutes such as Lachnospiraceae, Dorea, Coprococcus and Ruminococcus in CC receivers considerably reduced the differences between the gut microbial communities of mice reconstituted with faecal resuspensions of CC-treated and HFHS-treated donor mice (figure 5E, F and online supplementary table 7). The gut microbiota of HFHS and CC receivers was generally different from that of donor mice (figure $3 \mathrm{D}-\mathrm{G}$ ). The exceptions were the higher abundance of $A$. muciniphila and Barnesiella on days 1 and 2 postcolonisation, lower Lactobacillales on day 1 postcolonisation and higher Bifidobacterium on day 2 postcolonisation, traits seen in CC receivers and also observed in CC-treated donor mice. These data suggest that while short-term colonisation is not enough to establish a stable gut microbiota comparable to donor mice, a few taxa enriched in CC-treated mice faecal microbiota were sufficient to increase energy expenditure in our model.

We used qPCR to assess the abundance of key phylotypes harboured by CC-treated mice in faecal samples of HFHS and CC receivers. On day 1 postcolonisation, lower Lactobacillus spp as well as higher abundance of A. muciniphila were both found in the faeces of CC receivers when compared with HFHS receivers (figure $5 \mathrm{H}-\mathrm{Q}$ ). Interestingly, only $A$. muciniphila remained significantly enriched in the fæces of CC receivers in comparison with HFHS receivers on day 2 postcolonisation (figure 5Q). qPCR analysis did not confirm the changes in Bifidobacterium spp, Barnesiella spp, Allobaculum spp and Turicibacter spp found by $16 \mathrm{~S}$ sequencing analysis on days 1 and 2 postcolonisation in CC receivers versus HFHS receivers (figure 5I-K, N). The presence of Parabacteroides, Anaerostipes, Anaerotruncus and Roseburia was also not different during the first 2 days postcolonisation (figure $5 \mathrm{~L}, \mathrm{M}, \mathrm{O}, \mathrm{P}$ ). These results point towards A. muciniphila and Lactobacillus spp as being important drivers of increased energy expenditure and reduced weight gain on CC administration in diet-induced obese mice.

The remnant faecal slurries from CC-treated and vehicle-treated HFHS-fed donor mice were pooled together and the 


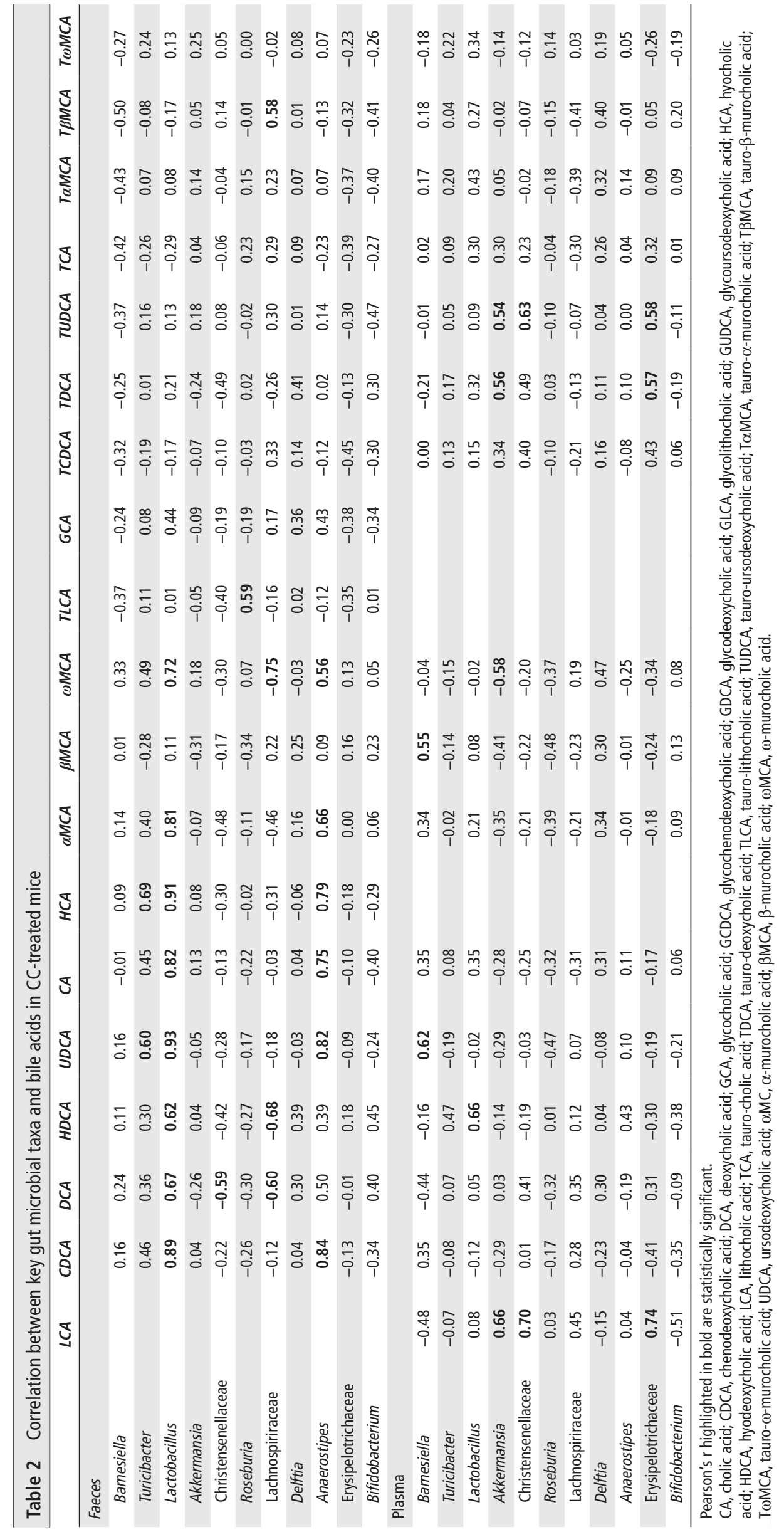



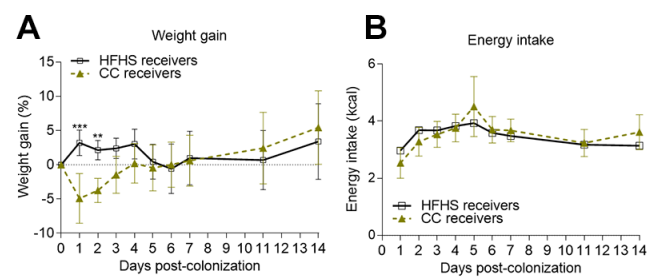

D

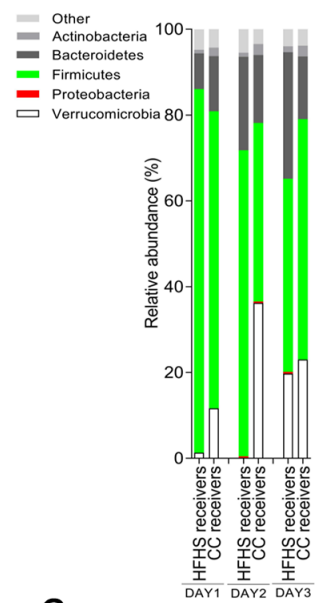

G FiB ratio
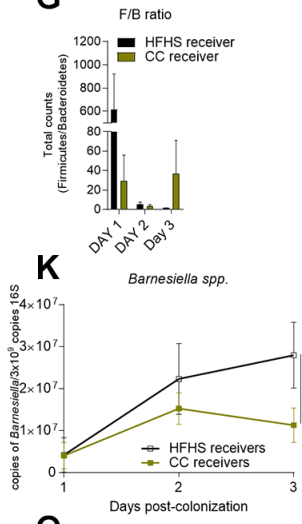

O Days post-colonization
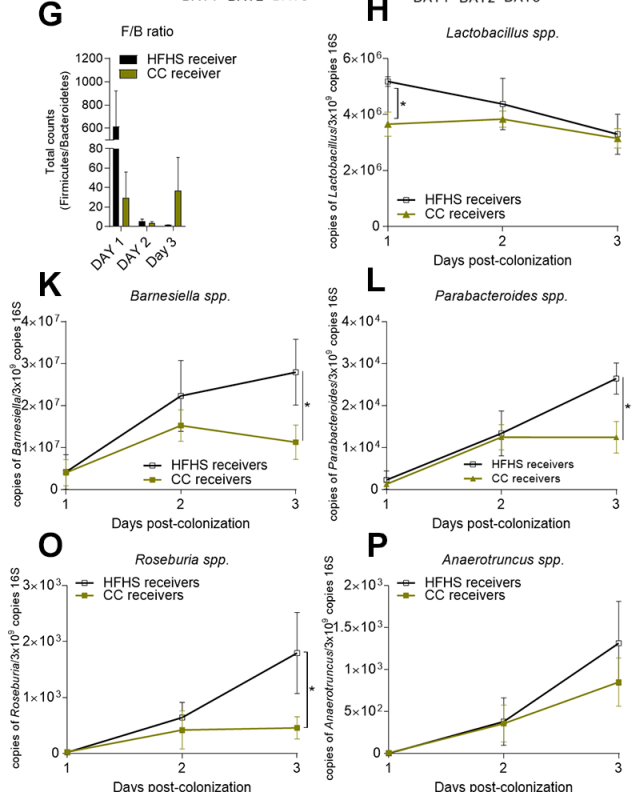

E

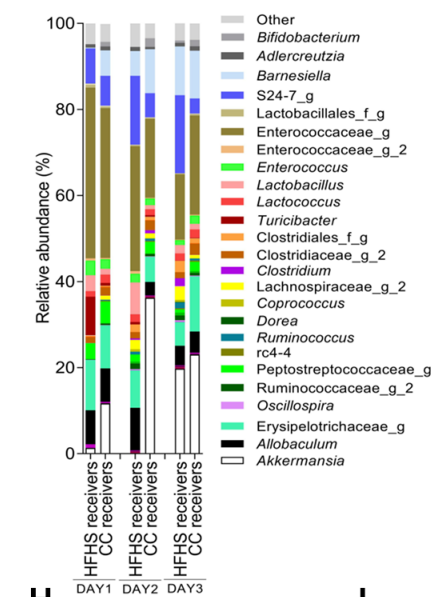

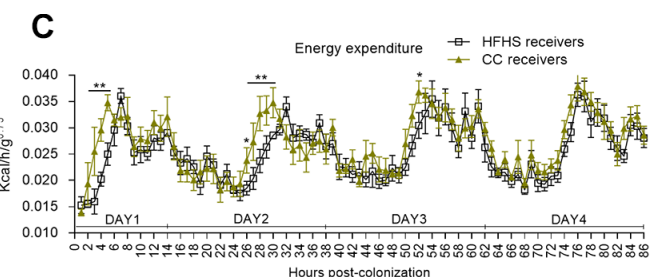

F

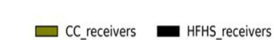

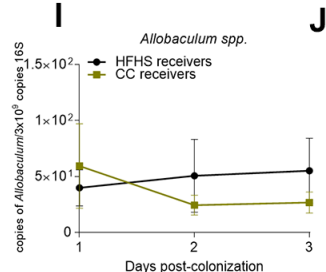
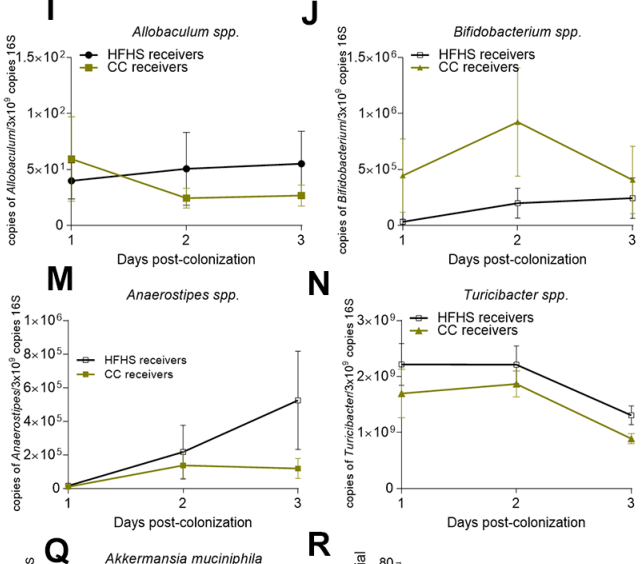

$\mathbf{N}$

Turicibacter spp.

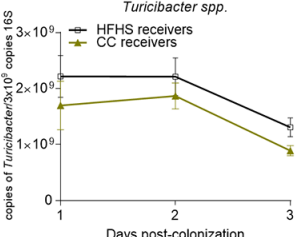

Figure 5 Reconstitution of germ-free mice with the faecal microbiota of camu camu (CC)-treated mice recapitulates the effects of CC administration on weight gain and energy expenditure. Germ-free mice were reconstituted either with the faecal slurry of high fat/high sucrose (HFHS)-fed vehicle-treated mice (HFHS receivers, $n=7$ ) or CC-treated mice (CC receivers, $n=7$ ) and kept on a low-fat diet in metabolic cages during the initial days postcolonisation. Mice were later transferred into sterile cages and kept under specific pathogen-free (SPF) conditions. (A) Weight gain; (B) energy intake; (C) energy expenditure (the relationship between metabolic rate and body mass was normalised by using the metabolic body size, ie, body mass ${ }^{0.75}$ ). (D, E) Taxonomic profile of HFHS and CC receivers at phylum and at the lowest taxonomic level achieved 1, 2 and 3 days postcolonisation (day $1-n=3$; day $2-n=5$; day $3-n=4$ ). (F) LEfSe analysis of taxa at the lowest taxonomic level possible that better discriminates the gut microbiota of HFHS and CC receivers at days 1, 2 and 3 postcolonisation. (G) Firmicutes to Bacteroidetes (F/B) ratio in HFHS and CC receivers (total counts Firmicutes/total counts Bacteroidetes). (H-Q) Taxa were quantified using qPCR and the number of copies of each taxon was normalised by $3 \times 10^{9}$ copies of $16 S$ (total bacteria). (R) Quantification by Folin-Cicalteu of total polyphenols present in the extract of CC and in faecal slurries used to colonise HFHS-receiver and CC-receiver mice. Data are expressed as the mean \pm SEM. Two-way repeated measures analysis of variance with a Bonferroni post hoc test was used to calculate the significance of the differences between time points. ${ }^{*} \mathrm{P}<0.05,{ }^{* *} \mathrm{p}<0.01$ and ${ }^{* * *} \mathrm{p}<0.001$.

total amount of polyphenols in these samples was assessed by Folin-Ciocalteu assay. As shown in figure $5 \mathrm{R}$, we found $4.58 \mathrm{mg}$ of polyphenols $/ 100 \mathrm{~g}$ of faeces in the resuspensions made from CC-treated stools. This concentration is $1430 \mathrm{x}$ lower than the polyphenolic concentration found in the CC extract (ie, $6550 \mathrm{mg} / 100 \mathrm{~g}$, figure $5 \mathrm{R}$ and table 1 ), strongly suggesting that the phenotype seen in CC receivers is not due to polyphenols present in the faecal resuspensions. 


\section{DISCUSSION}

We present evidence that daily treatment of HFHS-fed mice with a crude extract of CC containing a rich mixture of phytonutrients is sufficient to prevent diet-induced obesity and ameliorate the metabolic syndrome. Our work elucidates some key mechanisms underlying the beneficial action of CC treatment. The anti-obesity effect of CC is unrelated to changes in energy intake or excretion and rather attributed to increased energy expenditure and adaptive thermogenesis. CC treatment also restrained lipogenesis and activated lipolysis and free fatty acid oxidation in adipose tissues, which contributes to lower fat mass gain in these mice. Our data unravel a putative role for altered plasma BA pool size and composition that is consistent with brown fat activation and browning of subcutaneous white fat. Our findings suggest that changes in the gut microbiota are linked to key metabolic benefits of CC treatment. Interestingly, as depicted in online supplementary figure $1 \mathrm{j}, \mathrm{k}$, CC-treated mice became active earlier than vehicle-treated HFHS-fed mice, suggesting that alterations in the circadian cycle may also contribute to the metabolic benefits of CC.

CC-based products are widely marketed for their high content of vitamin $\mathrm{C}$ and their alleged strong anti-oxidant potential. ${ }^{13}$ We showed that mice treated daily with the same dose of vitamin C found in $200 \mathrm{mg} / \mathrm{kg}$ of CC (ie, $6.6 \mathrm{mg} / \mathrm{kg}$ ) displayed fat mass gain comparable to vehicle-treated HFHS-fed mice, strongly suggesting that vitamin $\mathrm{C}$, alone, is not causally involved in the anti-obesity effects of CC. CC extract is also rich in fibres, which possibly contributed to the benefits of CC treatment. However, increased faecal energy excretion (a typical trait of fibre-rich diets) was not seen in CC mice, suggesting a minor impact of fibres.

CC treatment prevented the HFHS-induced increase in BA pool size and composition. BA bind to FXR which in turn activates Shp to then inhibit their own synthesis in the liver and uptake in the ileum. FXR activation in the ileum also triggers the secretion of Fgf15, which contributes to the downregulation of BA synthesis in the liver through binding to Fgfr4. Our results suggest that the reduced BA pool size found in CC-treated HFHS mice likely contributes to downregulate FXR activity in the ileum of these mice. This conclusion is based on the lower mRNA levels of Shp in the ileum of CC mice, since the mRNA expression of Nr1h4 (encodes for FXR) poorly reflects its activity. ${ }^{24} 25$ Surprisingly, the hepatic mRNA expression of the rate-limiting enzyme involved in BA synthesis, Cyp7a1, along with the mRNA levels of Shp were not affected by $\mathrm{CC}$ administration. As we found a major decrease

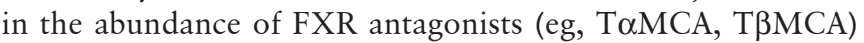
coupled with an increase in FXR agonists (eg, CDCA) in the BA pool of CC mice, we hypothesised that such a FXR-stimulatory BA profile might compensate for the inhibitory effect of reduced BA pool size, therefore disrupting the FXR-mediated negative feedback loop controlling BA synthesis in CC mice and keeping the levels of circulating BA lower than in vehicle-treated HFHS-fed mice. While future studies are warranted to fully decipher the mechanisms by which CC impacts BA homeostasis, it is of major interest that $\mathrm{CC}$ treatment reduced plasma BA profile in HFHS-fed mice.

Our results suggest that the plasma BA profile of CC mice may be relevant to enhance brown fat activation and browning of subcutaneous fat in CC mice and that the gut microbiota plays a role in this effect. The fact that Akkermansia is positively correlated with several BA in circulation is particularly interesting because CDCA and especially the secondary BA DCA and UDCA have been shown to act through TGR5 to potentiate the response of brown adipocytes to thyroid hormone and activate non-shivering thermogenesis in this tissue. ${ }^{21} \mathrm{We}$ also found a strong correlation between Ucp1 and TGR5 mRNA expression in the BAT of CC mice. Moreover, the gut hormone Fgf15 has been shown to trigger thermogenesis and browning of white fat depots by means of Fgfr4 activation. ${ }^{23}$ Consistently, we found a strong upregulation in the mRNA expression of Fgfr4 in the iWAT, suggesting that enhanced browning of subcutaneous white fat in CC mice is associated with increased responsiveness to Fgf15. Although the direct contribution of altered BA pool size and composition found in CC mice to enhance energy expenditure warrants further investigation, our study brings novel insights into the crosstalk BA-gut microbiota and its relationship with the anti-obesity effects of phytochemical-rich extracts.

Altered BA profile may contribute to reshape the gut microbiota in CC-treated mice. BA are antimicrobial molecules ${ }^{26}$ and the BA pool observed on high saturated fat feeding, which favours the presence of taurine-conjugated BA in the enterohepatic circulation, is associated with expansion of BA-resistant bacteria (eg, Bilophila wadsworthia and Lactobacillus spp) in the gut environment. ${ }^{24} 27$ The reduction of BA pool size found in CC mice may remove the competitive advantage of Lactobacillus spp and contribute to mitigate their presence in the gut. The likely increment in the antioxidant potential in the lumen associated with the intake of CC phytochemicals may restrain oxygen availability and further compromise the bloom of facultative anaerobe species such as Lactobacilli. This hypothesis is supported by a major reduction in Lactobacillus spp abundance observed in mice treated with the antioxidant tempol. ${ }^{24}$

CC markedly increased A. muciniphila population in the gut microbiota of diet-induced obese mice. This is in line with several reports showing the metabolic benefits of A. muciniphila. ${ }^{28} 29$ Furthermore, we and others have previously shown the association between dietary proanthocyanidin (PAC)-rich berry extracts and a prebiotic-like effect on A. muciniphila. ${ }^{9} 1030$ As previously reported, ${ }^{13}$ our analysis showed a remarkable concentration of galloylated PACs in the CC extract, pointing to an effect of PACs on A. muciniphila. CC is also rich in ellagitannins, and while ellagitannin-rich extracts seem to inhibit the growth of $A$. muciniphila in vitro, this bacterium seems capable of degrading ellagitannins and ellagic acid, which could provide in vivo a competitive advantage to A. muciniphila. ${ }^{31}$ In humans, however, intake of ellagitannin-rich pomegranate juice did not increase the presence of A. muciniphila, suggesting that other polyphenols and the pre-existing abundance of A. muciniphila are important factors. ${ }^{32} 33$ While the understanding of how polyphenols and other phytonutrients modify the gut microbial environment to favour the expansion of $A$. muciniphila warrants further investigation, the interplay between A. muciniphila and $\mathrm{BA}$ is also largely unknown. A survey in the KEGG database showed absence of pathways linked to BA deconjugation/dehydroxylation in the genome of A. muciniphila, but this microbe occupies a very specific niche in the gut environment, which may confer resistance to the antimicrobial effect of certain BA. In vitro experiments are warranted to test the ability of this bacterium to transform and resist to BA.

Faecal transplant experiments revealed that the gut microbiota of CC-treated mice can transiently and partially recapitulate in germ-free receiver mice the phenotype of conventional mice treated with CC. Receiver mice were fed a low-fat diet to remove a major obesogenic pressure and therefore allow for a quantifiable effect of FMT on body weight in the short-term. 
It should also be mentioned that faecal resuspensions contained only negligible amounts of polyphenols, which points to an effect of bacteria and/or microbial molecules. While our colonisation approach did not establish in CC-receiver mice a stable gut microbiota comparable to that of CC donor mice, coupled $16 \mathrm{~S}$-based sequencing and qPCR analysis suggest that a few key taxa are sufficient to increase energy expenditure and reduce weight gain. A. muciniphila is likely a major contributor since its abundance is as high as $11.6 \%$ and $36.22 \%$ in the first and second days postcolonisation, respectively. This is supported by previous studies showing that the oral administration of $A$. muciniphila to diet-induced obese mice counteracted obesity along with other features of the metabolic syndrome. ${ }^{28} 2934$ However, the abundance of A. muciniphila is also high in the third day postcolonisation, when oxygen consumption and body weight change became similar between CC-receiver and HFHS-receiver mice, indicating that other gut microbial characteristics, such as lower Lactobacillus and higher Bifidobacterium, may also play a role. This would be in line with previous studies showing positive associations between Lactobacillus and obesity $^{35-37}$ and negative correlations between Bifidobacterium and obesity. ${ }^{3638}$

Acknowledgements The authors would like to thank Christine Dion, Christine Dallaire, Valérie Dumais and Joanie Dupont-Morissette for their expert help with animal care.

Contributors FFA, GP and AM conceived the study. FFA, MLB, TVV, JT, SD and RTN performed the experiments. FFA wrote the manuscript, prepared the figures and was responsible for data compilation and integration. All authors contributed to discuss the results and to research directions. All authors approved the manuscript.

Funding This work was funded by the Ministère du Développement Économique, de I'Innovation et de I'Exportation (MDEIE, PSR-SIIRI-444), J.A. deSève Foundation, by a joint grant from Fundação de Amparo à Pesquisa do Estado de São Paulo (FAPESP)-Agence Universitaire de la Francophonie (AUF) and by a Canadian Institutes of Health Research (CIHR) foundation grant (FDN\#143247) to AM.

Competing interests None declared.

Patient consent Not required.

Ethics approval Laval University Animal Ethics Committee.

Provenance and peer review Not commissioned; externally peer reviewed.

Data sharing statement All raw sequences from 16S rRNA gene-based analysis have been deposited in the public European Nucleotide Archive server under accession number PRJEB23031. Data sets generated and analysed during this study are available from the corresponding author on reasonable request.

\section{REFERENCES}

1 Finucane MM, Stevens GA, Cowan MJ, et al. National, regional, and global trends in body-mass index since 1980: systematic analysis of health examination surveys and epidemiological studies with 960 country-years and 9.1 million participants. The Lancet 2011;377:557-67.

2 Kelly T, Yang W, Chen CS, et al. Global burden of obesity in 2005 and projections to 2030. Int J Obes 2008;32:1431-7.

3 Olshansky SJ, Passaro DJ, Hershow RC, et al. A potential decline in life expectancy in the United States in the 21st century. N Engl J Med 2005;352:1138-45.

4 Ridaura VK, Faith JJ, Rey FE, et al. Gut microbiota from twins discordant for obesity modulate metabolism in mice. Science 2013;341:1241214.

5 Caesar R, Tremaroli V, Kovatcheva-Datchary P, et al. Crosstalk between Gut Microbiota and Dietary Lipids Aggravates WAT Inflammation through TLR Signaling. Cell Metab 2015;22:658-68.

6 David LA, Maurice CF, Carmody RN, et al. Diet rapidly and reproducibly alters the human gut microbiome. Nature 2014;505:559-63.

7 Zhernakova A, Kurilshikov A, Bonder MJ, et al. Population-based metagenomics analysis reveals markers for gut microbiome composition and diversity. Science 2016:352:565-9.

8 Falony G, Joossens M, Vieira-Silva S, et al. Population-level analysis of gut microbiome variation. Science 2016;352:560-4.

9 Anhê FF, Roy D, Pilon G, et al. A polyphenol-rich cranberry extract protects from diet-induced obesity, insulin resistance and intestinal inflammation in association with increased Akkermansia spp. population in the gut microbiota of mice. Gut 2015;64:872-83.

10 Roopchand DE, Carmody RN, Kuhn P, et al. Dietary polyphenols promote growth of the gut bacterium akkermansia muciniphila and attenuate high-fat diet-induced metabolic syndrome. Diabetes 2015;64:2847-58.

11 Bataglion GA, da Silva FMA, Eberlin MN, et al. Determination of the phenolic composition from Brazilian tropical fruits by UHPLC-MS/MS. Food Chem 2015;180:280-7.

12 Langley PC, Pergolizzi JV, Taylor R, et al. Antioxidant and associated capacities of Camu camu (Myrciaria dubia): a systematic review. J Altern Complement Med 2015;21:8-14.

13 Fracassetti D, Costa C, Moulay L, et al. Ellagic acid derivatives, ellagitannins, proanthocyanidins and other phenolics, vitamin C and antioxidant capacity of two powder products from camu-camu fruit (Myrciaria dubia). Food Chem 2013:139:578-88.

14 Feldmann HM, Golozoubova V, Cannon B, et al. UCP1 ablation induces obesity and abolishes diet-induced thermogenesis in mice exempt from thermal stress by living at thermoneutrality. Cell Metab 2009;9:203-9.

15 Helppi J, Schreier D, Naumann $\mathrm{R}$, et al. Mouse reproductive fitness is maintained up to an ambient temperature of $28^{\circ} \mathrm{C}$ when housed in individually-ventilated cages. Lab Anim 2016;50:254-63.

16 Kleiber M. Body size and metabolic rate. Physiol Rev 1947;27:511-41.

$17 \mathrm{Kim} \mathrm{KW}$, Donato J, Berglund ED, et al. FOXO1 in the ventromedial hypothalamus regulates energy balance. J Clin Invest 2012;122:2578-89.

18 Cotillard A, Kennedy SP, Kong LC, et al. Dietary intervention impact on gut microbial gene richness. Nature 2013;500:585-8.

19 Le Chatelier E, Nielsen T, Qin J, et al. Richness of human gut microbiome correlates with metabolic markers. Nature 2013:500:541-6.

20 Yasir M, Angelakis E, Bibi F, et al. Comparison of the gut microbiota of people in France and Saudi Arabia. Nutr Diabetes 2015;5:e153.

21 Watanabe M, Houten SM, Mataki C, et al. Bile acids induce energy expenditure by promoting intracellular thyroid hormone activation. Nature 2006;439:484-9.

22 Thomas C, Gioiello A, Noriega L, et al. TGR5-mediated bile acid sensing controls glucose homeostasis. Cell Metab 2009;10:167-77.

23 Fang S, Suh JM, Reilly SM, et al. Intestinal FXR agonism promotes adipose tissue browning and reduces obesity and insulin resistance. Nat Med 2015:21:159-65.

24 Li F, Jiang C, Krausz KW, et al. Microbiome remodelling leads to inhibition of intestinal farnesoid $X$ receptor signalling and decreased obesity. Nat Commun 2013;4:2384.

25 Watanabe M, Horai Y, Houten SM, et al. Lowering bile acid pool size with a synthetic farnesoid $X$ receptor (FXR) agonist induces obesity and diabetes through reduced energy expenditure. J Biol Chem 2011;286:26913-20.

26 Ridlon JM, Kang DJ, Hylemon PB, et al. Bile acids and the gut microbiome. Curr Opin Gastroenterol 2014:30:332-8

27 Devkota S, Wang Y, Musch MW, et al. Dietary-fat-induced taurocholic acid promotes pathobiont expansion and colitis in II10-/- mice. Nature 2012;487:104-8.

28 Plovier $\mathrm{H}$, Everard A, Druart C, et al. A purified membrane protein from Akkermansia muciniphila or the pasteurized bacterium improves metabolism in obese and diabetic mice. Nat Med 2017;23:107-13.

29 Everard A, Belzer C, Geurts L, et al. Cross-talk between Akkermansia muciniphila and intestinal epithelium controls diet-induced obesity. Proc Natl Acad Sci U S A 2013;110:9066-71

30 Anhê FF, Varin TV, Le Barz M, et al. Gut microbiota dysbiosis in obesity-linked metabolic diseases and prebiotic potential of polyphenol-rich extracts. Curr Obes Rep 2015:4:389-400.

31 Henning SM, Summanen PH, Lee RP, et al. Pomegranate ellagitannins stimulate the growth of Akkermansia muciniphila in vivo. Anaerobe 2017:43:56-60.

32 González-Sarrías A, García-Villalba R, Romo-Vaquero M, et al. Clustering according to urolithin metabotype explains the interindividual variability in the improvement of cardiovascular risk biomarkers in overweight-obese individuals consuming pomegranate: A randomized clinical trial. Mol Nutr Food Res 2017;61:1600830.

33 Mosele Jl, Gosalbes MJ, Macià A, et al. Effect of daily intake of pomegranate juice on fecal microbiota and feces metabolites from healthy volunteers. Mol Nutr Food Res 2015; 59:1942-53.

34 Anhê FF, Marette A. A microbial protein that alleviates metabolic syndrome. Nat Med 2017:23:11-12.

35 Khan $M$, Raoult $D$, Richet $H$, et al. Growth-promoting effects of single-dose intragastrically administered probiotics in chickens. Br Poult Sci 2007:48:732-5.

36 Million M, Maraninchi M, Henry $M$, et al. Obesity-associated gut microbiota is enriched in Lactobacillus reuteri and depleted in Bifidobacterium animalis and Methanobrevibacter smithii. Int J Obes 2012;36:817-25.

37 Armougom F, Henry M, Vialettes B, et al. Monitoring bacterial community of human gut microbiota reveals an increase in Lactobacillus in obese patients and Methanogens in anorexic patients. PLoS One 2009;4:e7125.

38 An HM, Park SY, Lee DK, et al. Antiobesity and lipid-lowering effects of Bifidobacterium spp. in high fat diet-induced obese rats. Lipids Health Dis 2011;10:116. 